\title{
The Incidence of Peripheral Catheter-Related Thrombosis in Surgical Patients
}

\author{
Amy Leung, ${ }^{1,2,3}$ Clare Heal, ${ }^{1}$ Jennifer Banks, ${ }^{1}$ Breanna Abraham, \\ Gian Capati, ${ }^{2}$ and Casper Pretorius ${ }^{1,2}$ \\ ${ }^{1}$ James Cook University, School of Medicine and Dentistry, 4740 Mackay, QLD, Australia \\ ${ }^{2}$ Mackay Base Hospital, Mackay, QLD 4650, Australia \\ ${ }^{3}$ Mater Health Services, Corner of Raymond Terrace and Stanley Street, South Brisbane, QLD 4101, Australia \\ Correspondence should be addressed to Amy Leung; amy.leung@my.jcu.edu.au
}

Received 3 October 2015; Accepted 16 December 2015

Academic Editor: Domenico Prisco

Copyright (C) 2016 Amy Leung et al. This is an open access article distributed under the Creative Commons Attribution License, which permits unrestricted use, distribution, and reproduction in any medium, provided the original work is properly cited.

\begin{abstract}
Background. Central venous catheters and peripherally inserted central catheters are well established risk factors for upper limb deep vein thrombosis. There is limited literature on the thrombosis rates in patients with peripheral catheters. A prospective observational study was conducted to determine the incidence of peripheral catheter-related thrombosis in surgical patients. Methods. Patients deemed high risk for venous thrombosis with a peripheral catheter were considered eligible for the study. An ultrasound was performed on enrolment into the study and at discharge from hospital. Participants were reviewed twice a day for clinical features of upper limb deep vein thrombosis during their admission and followed up at 30 days. Results. 54 patients were included in the study. The incidence of deep vein thrombosis and superficial venous thrombosis was $1.8 \%$ and $9.2 \%$, respectively. All cases of venous thrombosis were asymptomatic. Risk factor analysis was limited by the low incidence of thrombosis. Conclusion. This study revealed a low incidence of deep vein thrombosis in surgical patients with peripheral catheters (1.8\%). The study was underpowered; therefore the association between peripheral catheters and thrombosis is unable to be established. Future studies with larger sample sizes are required to determine the association between peripheral catheters and thrombosis.
\end{abstract}

\section{Introduction}

Upper limb deep vein thrombosis (DVT) refers to the formation of a fibrin clot within the subclavian, axillary, and brachial veins of the arm [1]. The vast majority of cases of upper limb DVT are associated with the use of indwelling intravenous catheters [2]. While any intravenous catheter has the potential to lead to venous thrombosis, the literature has mainly focused on the thrombosis rates of central venous catheters (CVCs) and peripherally inserted central catheters (PICCs) [3]. Over 330 million peripheral catheters are placed every year in America alone, required by up to $70 \%$ of patients in hospital. Peripheral catheters are often used in surgical patients for the administration of intravenous fluids and intravenous medications and for anaesthetic purposes [4]. Although the insertion of a peripheral catheter is the most common invasive procedure in hospitals, there is a paucity of data regarding thrombosis rates in patients with peripheral catheters. As many surgical patients require a peripheral catheter during their admission, we have conducted a prospective study to detect the incidence of peripheral catheter-related DVT in the general surgical unit and the potential associated risk factors. A secondary aim was to determine the incidence of catheter-related superficial vein thrombosis (SVT).

\section{Methods}

2.1. Eligible Patients. All patients hospitalized in the general surgical unit classified as high risk for venous thromboembolism (VTE) with a peripheral intravenous catheter in the dorsal hand or forearm were considered for inclusion. We accepted peripheral intravenous catheters of any gauge. We defined high risk as age over 65 years and one other risk factor 
either an underlying malignancy, thrombophilia (Factor C deficiency, Factor $\mathrm{S}$ deficiency, Factor 5 Leiden mutation, antithrombin 3 deficiency, antiphospholipid syndrome, and prothrombin 20210A mutation), or recent major surgery according to criteria from the Royal Australasian College of Surgeons (RACS) [5]. Exclusion criteria included previous or current upper limb or chest trauma, previous or current upper limb, neck, or axillary surgery, previous or current upper limb DVT, previous mastectomy, thoracic outlet syndrome, enlarged lymph nodes/tumours causing compression, or current central venous catheter or peripherally inserted central catheter. Participants were prospectively followed up for 30 days from enrolment.

2.2. Study Design. The study was a prospective observational single arm cohort study conducted in the general surgical unit of Mackay Base Hospital. The study period occurred from August 2014 to July 2015. The study received ethical approval from the Townsville Hospital and Health Services Human Resources and Ethics Committee on the 30th of May 2014. Informed consent was obtained from all individual participants included in the study. On enrolment, the participant's demographics, medical comorbidities, full blood count, international normalised ratio of prothrombin time of blood coagulation (INR), and catheter characteristics were collected. All patients had an ultrasound examination of the superficial and deep veins of the cannulated arm on enrolment into the study and at discharge. Colour duplex sonography was selected for diagnosis of DVT as it has a high sensitivity (93\%) and specificity (93\%) and is noninvasive and easy to perform [6]. All ultrasounds were performed by the sonographers at the Mackay Base Hospital Radiology Department. The criteria for upper extremity thrombosis were defined as the presence of a thrombus, lack of flow, nonpulsatile and nonphasic flow, and lack of compressibility of the veins [7]. DVT was classified as thrombosis of the subclavian, axillary, or brachial veins. Thrombosis of the cephalic or basilic vein was classified as a SVT. During the admission period, patients were reviewed twice a day for signs and symptoms of upper limb DVT. Patients were asked if they had experienced upper limb pain and swelling. On examination, abnormal vital signs, tenderness, oedema, and erythema of the cannulated arm were noted. All patients were followed up at thirty days with either a phone consult or outpatient appointment to review symptoms of venous thromboembolism (VTE).

2.3. Statistical Analysis. The incidence of peripheral catheterrelated DVT has only been reported in one previous study. Therefore we estimated a $20 \%$ prevalence based on the results of this study. Based on the estimated prevalence, we calculated a sample size of 62 participants for $95 \%$ Confidence Intervals (CI) of $10 \%$ to $30 \%$. The incidence of peripheral catheter-related thrombosis is reported as the proportion of enrolled patients who had an ultrasound confirmed thrombus. Corresponding 95\% CIs were calculated for the proportions of thrombosis. Descriptive statistics were generated for all included cases. Continuous variables were expressed as medians and interquartile ranges (IQR 25th and 75th percentile) or means and standard deviations (SD). The categorical variables were expressed as numbers and percentages. Bivariate analysis was performed using Fisher's exact test with thrombosis as the dependent variable and various risk factors as the independent variable. The data was analysed using SPSS version 20 (SPSS Inc., Chicago, IL, USA) for Windows; statistical significance was given to $P$ values $<0.05$.

\section{Results}

From August 2014 to July 2015 there were 337 participants aged 65 years and over who had a peripheral catheter placed in the dorsal hand or forearm in the general surgical unit. Two-hundred and sixty patients did not meet eligibility criteria and 17 declined to enter the study. Sixty patients consented to participate in the study. Six patients did not complete the study. Five patients were lost to follow-up and one patient passed away from an underlying medical condition unrelated to the study. In total, data from 54 patients was analysed. The participant characteristics are listed in Table 1 . None of the recruited participants had a thrombophilia. The incidence of upper limb DVT was 1.8\% (1 out of 54 patients, 95\% CI $-1.7 \%$ to $5.4 \%$ ). The thrombus was located in the brachial vein. The only risk factors the patient had were age over 65 years and recent surgery. The patient was commenced on six months of warfarin as per the physicians at Mackay Base Hospital. The incidence of SVT was 9.25\% (5 out of 54 patients, 95\% CI 1.5\% to $17 \%$ ). Four were located in the cephalic vein and one in the basilic vein. All patients with SVT were treated with 30 days of therapeutic low molecular weight heparin (LMWH) as per the American College of Chest Physician Guidelines for antithrombotic therapy for venous thromboembolic disease [8]. The characteristics of the participants with peripheral catheter-related venous thrombosis is displayed in Table 2. The overall incidence of thrombotic events was $11.1 \%$ (95\% CI $2.7 \%$ to $19.5 \%)$. All cases of venous thrombosis were asymptomatic. There were no cases of clinically detected pulmonary embolism. None of the risk factors investigated were found to be statistically significant for association with upper extremity thrombosis, as listed in Table 3. At the 30-day follow-up there were no reported adverse events.

\section{Discussion}

Ours is the first prospective study designed to detect the incidence of peripheral catheter-related DVT and to identify associated risk factors in surgical patients. We detected a low incidence of peripheral catheter-DVT (incidence of 1.8\%) with no associated risk factors. However there was a higher incidence of peripheral catheter-related SVT (9.8\%). All cases of peripheral catheter-related thrombosis in our study were asymptomatic and detected on ultrasound as a result of our study. There were no cases of pulmonary embolism clinically detected in our study.

The low incidence of peripheral catheter-related DVT reinforces the results of a previous study. A randomised control trial comparing the efficacy of PICCS and peripheral 
TABLE 1: Characteristics of the participants.

\begin{tabular}{|c|c|}
\hline Variable & Result \\
\hline Age (years), median (IQR) & $73(69-79.25)$ \\
\hline Gender, $n(\%)$ female & $21(38.9 \%)$ \\
\hline \multicolumn{2}{|l|}{ Ethnicity, $n(\%)$} \\
\hline Caucasian & $53(98.1 \%)$ \\
\hline Aboriginal and Torres Strait Islander & $1(1.98 \%)$ \\
\hline Anticoagulation, $n(\%)$ & $25(46.3 \%)$ \\
\hline Haemaglobin, median (IQR) & $130(121.75-142.25)$ \\
\hline Platelets, median (IQR) & $226(177-316.5)$ \\
\hline INR, median (IQR) & $1.1(1.0-1.1)$ \\
\hline \multicolumn{2}{|l|}{ Catheter size, $n(\%)$} \\
\hline $18 \mathrm{G}$ & $48(88.9 \%)$ \\
\hline $20 \mathrm{G}$ & $3(5.6 \%)$ \\
\hline $22 \mathrm{G}$ & $3(5.6 \%)$ \\
\hline $\begin{array}{l}\text { Time of catheterization in days, } \\
\text { median (IQR) }\end{array}$ & $3.83(2-5)$ \\
\hline \multicolumn{2}{|l|}{ Location of peripheral catheter, $n(\%)$} \\
\hline Dorsum hand & $17(31.5 \%)$ \\
\hline Wrist & $6(11.1 \%)$ \\
\hline Forearm & $10(18.5 \%)$ \\
\hline Cubital fossa & $21(38.9 \%)$ \\
\hline Resited peripheral catheters, $n(\%)$ & $52(96.3 \%)$ \\
\hline $\begin{array}{l}\text { Number of attempts at catheterization, } \\
\text { mean (SD) }\end{array}$ & $1(0.79)$ \\
\hline BMI, $n(\%)$ & $17(31.5 \%)$ \\
\hline Diabetes, $n(\%)$ & $15(27.8 \%)$ \\
\hline Malignancy, $n(\%)$ & $20(37 \%)$ \\
\hline Metastatic disease, $n(\%)$ & $3(5.6 \%)$ \\
\hline Recent surgery, $n(\%)$ & $40(74.1 \%)$ \\
\hline
\end{tabular}

catheters found a $3.4 \%$ incidence of peripheral catheterrelated DVT. Similar to our study ultrasound was performed at the beginning and end of catheterization. There was a higher incidence of peripheral catheter-related SVT than the incidence of peripheral catheter-related DVT in our study. The incidence of peripheral catheter-related SVT was lower than figures reported in a previous study $(9.2 \%$ compared to $44 \%$ ). The increased incidence of SVT in Periard's study is likely attributed to larger gauge of catheters used and increased time of catheterization [9]. The thrombogenicity of peripheral catheters can be attributed to endothelial injury and venous stasis, two of the components of Virchow's triad of thrombosis. The endothelium is often damaged during the insertion of a peripheral catheter [3]. In addition, the presence of a peripheral catheter in a vein reduces blood flow and potentially causes venous stasis. Peripheral catheters are often inserted into the smaller superficial veins of the upper limb creating a smaller catheter-to-vein ratio. Catheter-tovein ratio has been suggested to be a contributing factor to thrombosis due to increased risk of stasis from a slower flow. This is evident in studies with PICC-lines as PICCs inserted into the smaller cephalic veins are more likely to thrombose [10].

We assessed recent surgery, malignancy, metastatic disease, increased BMI, diabetes mellitus, cannula gauge, number of attempts at cannulation, and length of catheterization for association with catheter-related thrombosis. None of the risk factors assessed in our study were found to have a significant association with catheter-related thrombosis. However, the data analysis was limited by the small number of events. In our study $83 \%$ of participants with upper extremity thrombosis had recent surgery. Previous studies report mixed results regarding recent surgery as a risk factor for catheter-related thrombosis [11-15]. Wilson et al. demonstrated that surgery $>1$ hour in length was a risk factor for catheter-related thrombosis in a prospective study. The development of a thrombus was attributed to venous stasis from prolonged paresis of the arm during surgery [15]. Half of the patients with a peripheral catheter-related thrombosis had a malignancy. The association between malignancy and venous thrombosis has been well established. Malignancy is believed to induce a hypercoagulable state through activation of the coagulation cascade and generation of thrombin [11].

The results of our study raise questions regarding screening for asymptomatic upper extremity thrombosis. None of the patients with peripheral catheter-related thrombosis were symptomatic. Previous studies have reported similar findings where all cases of thrombosis were found incidentally on ultrasound [9]. These results confirm the need for imaging in the diagnosis of peripheral catheter-related thrombosis as most cases are asymptomatic.

There are strong arguments in literature regarding anticoagulation of symptomatic upper limb DVT as it prevents propagation of the thrombus and development of a pulmonary embolism (PE) [9]. The clinical significance of an asymptomatic upper limb DVT is poorly documented as the majority of studies are conducted on symptomatic patients [16]. As the nature of asymptomatic upper limb DVT is unknown, the risks and benefits of treatment are unknown. Theoretically the risk of embolization of the asymptomatic thrombus should be the same as a symptomatic upper limb DVT. In fact, asymptomatic DVT of the lower limb has long been regarded as a source of embolization. In a large autopsy series lower limb DVT was detected in $83 \%$ of patients who died from PE; however only 19\% were symptomatic [17]. The findings of the study suggest that asymptomatic upper limb DVT may be an overlooked source of PE. However large randomised studies would be required to determine the clinical significance of an asymptomatic upper limb DVT.

We detected five cases of SVT in our study. SVT has previously been regarded as a benign disease; however there is a growing body of evidence to suggest otherwise [18, 19]. Decousus et al. demonstrated that $25 \%$ of patients with a lower limb superficial venous thrombosis developed a concomitant deep vein thrombosis and 3.9\% had a symptomatic pulmonary embolism [20]. While there are currently no studies regarding the clinical significance of an upper extremity DVT, based on the studies conducted on the lower limb we decided to treat patients diagnosed with SVT with 


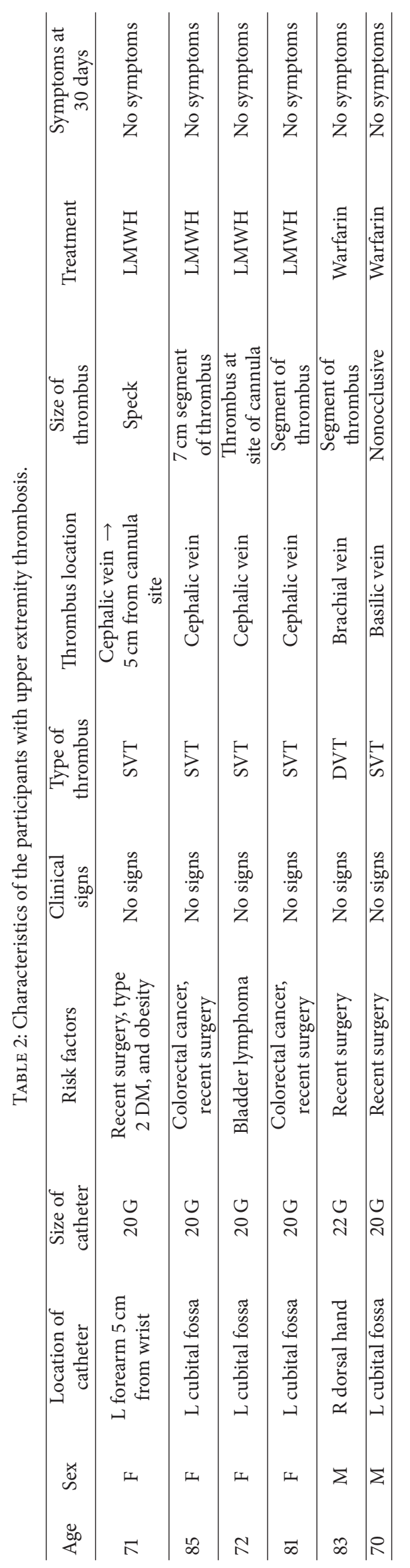


TABLE 3: Risk factors.

\begin{tabular}{lcc}
\hline Risk factor & Number of thromboses & $P$ value \\
\hline Gender (female) & $4 / 21(19 \%)$ & 0.193 \\
Ethnicity (Caucasian) & $6 / 53(11.3 \%)$ & 1 \\
Diabetes & $1 / 15(6.66 \%)$ & 1 \\
Metastatic disease & $0 / 3(0 \%)$ & 1 \\
Anticoagulation & $3 / 25(12 \%)$ & 1 \\
Elevated WCC & $1 / 8(12.5 \%)$ & 1 \\
BMI $>30$ & $2 / 17(11.76 \%)$ & 1 \\
Cannula LOS $>1$ & & NS \\
Size of cannula & & NS \\
Cubital fossa & $4 / 21(19 \%)$ & NS \\
More than 1 attempt & $1 / 12(8.33 \%)$ & NS \\
Malignancy & $3 / 20(15 \%)$ & 0.659 \\
Surgery & $4 / 40(10 \%)$ & 0.643 \\
\hline
\end{tabular}

30 days of LMWH. At the 30-day follow-up there were no adverse events or complications reported.

There were no cases of clinically detected PE in our study. Similar findings have been reported in previous studies. In a large retrospective series, Chemaly et al. reported an incidence of $3.4 \%$ of PE in patients with PICC-lines [21]. The incidence of asymptomatic $\mathrm{PE}$ is higher in patients with catheter-related thrombosis, with up to $17 \%$ of patients with catheter-related thrombosis having an asymptomatic PE [22]. However the mortality rate associated with asymptomatic $\mathrm{PE}$ appears to be low. It is uncertain whether investigation of asymptomatic PE would be of benefit considering the invasiveness of the investigations required [23].

There are several limitations to our study. The present study is a prospective observational study conducted in a single regional institution; therefore the findings of this study may not be applicable to other hospital populations. There is inherent bias associated with the observational design of the study as confounders are unable to be controlled and there are a lack of internal controls. The study was underpowered; therefore the confidence intervals are very broad, indicating that the results are imprecise. The low incidence of peripheral catheter-related thrombosis limited the ability to identify associated risk factors. Nonconsecutive patients were enrolled into the study; therefore the study sample may not be a true reflection of the intended sample. Ideally, both arms would be scanned for a DVT; however this would have been too difficult considering financial constraints of the study. Furthermore patients were only followed up for 30 days; therefore symptoms of upper limb DVT after 30 days are unknown.

Despite the limitations, there were also several strengths with this study. This is the first study to investigate the incidence of peripheral catheter-related venous thrombosis in surgical patients. The prospective design and investigation of all study participants prevented missing any case of peripheral catheter-related thrombosis. In addition this confirmed the asymptomatic nature of peripheral catheterrelated thrombosis. We also used objective investigations to diagnose thrombosis preventing misdiagnosis. This is also the first study to investigate the risk factors for upper limb superficial venous thrombosis.

\section{Conclusion}

In our study we recorded a low incidence of peripheral catheter-related DVT $(1.8 \%, 95 \%$ CI $-1.7 \%$ to $5.4 \%)$, but a higher incidence of superficial venous thrombosis $(9.25 \%$, 95\% CI 1.5\% to 17\%). The small sample size and low incidence of peripheral catheter-related thrombosis limited our ability to determine the association between peripheral catheters and upper limb DVT. Future studies will be required to show the natural history of an asymptomatic upper limb DVT through regular ultrasounds and investigation for occult pulmonary emboli.

\section{Ethical Approval}

All procedures performed in studies involving human participants were in accordance with the ethical standards of the institutional and/or national research committee and with the 1964 Helsinki declaration and its later amendments or comparable ethical standards.

\section{Conflict of Interests}

None of the authors have any conflict of interests.

\section{Acknowledgments}

The authors would like to acknowledge the assistance of Dr. Antonio Pace for reporting the ultrasounds and the sonographers at Mackay Base Hospital (Susanna Rae, Ainslie Heinke, Heidi Croxson and Amy Leigh). Funding for the project was obtained from the Private Practice Trust Fund and James Cook University School of Medicine and Dentistry.

\section{References}

[1] J. D. Grant, S. M. Stevens, S. C. Woller et al., "Diagnosis and management of upper extremity deep-vein thrombosis in adults," Thrombosis and Haemostasis, vol. 108, no. 6, pp. 10971108, 2012.

[2] N. Kucher, "Deep-vein thrombosis of the upper extremities," The New England Journal of Medicine, vol. 364, no. 9, pp. 861869,2011

[3] H. V. Joffe, N. Kucher, V. F. Tapson, and S. Z. Goldhaber, "Upperextremity deep vein thrombosis: a prospective registry of 592 patients," Circulation, vol. 110, no. 12, pp. 1605-1611, 2004.

[4] W. Zingg and D. Pittet, "Peripheral venous catheters: an under-evaluated problem," International Journal of Antimicrobial Agents, vol. 34, supplement 4, pp. S38-S42, 2009.

[5] Royal Australasian College of Surgeons, Procedure list for SET and IMG General Surgery, Royal Australasian College of Surgeons, Melbourne, Australia, 2015, http://www.surgeons.org/ for-health-professionals/audits-and-surgical-research/morbidity-audit-and-logbook-tool/general-surgery/.

[6] M. Di Nisio, G. L. Van Sluis, P. M. M. Bossuyt, H. R. Büller, E. Porreca, and A. W. S. Rutjes, "Accuracy of diagnostic tests for 
clinically suspected upper extremity deep vein thrombosis: a systematic review," Journal of Thrombosis and Haemostasis, vol. 8, no. 4, pp. 684-692, 2010.

[7] H. J. Baarslag, M. M. W. Koopman, J. A. Reekers, and E. J. R. van Beek, "Diagnosis and management of deep vein thrombosis of the upper extremity: a review," European Radiology, vol. 14, no. 7, pp. 1263-1274, 2004.

[8] C. Kearon, S. R. Kahn, G. Agnelli, S. Goldhaber, G. E. Raskob, and A. J. Comerota, "Antithrombotic therapy for venous thromboembolic disease. American College of Chest Physicians evidence-based clinical practice guidelines (8th edition)," Chest, vol. 133, no. 6, pp. 454-545, 2008.

[9] D. Periard, P. Monney, G. Waeber et al., "Randomized controlled trial of peripherally inserted central catheters vs. peripheral catheters for middle duration in-hospital intravenous therapy," Journal of Thrombosis and Haemostasis, vol. 6, no. 8, pp. 1281-1288, 2008.

[10] T. P. Nifong and T. J. McDevitt, "The effect of catheter to vein ratio on blood flow rates in a simulated model of peripherally inserted central venous catheters," Chest, vol. 140, no. 1, pp. 4853, 2011.

[11] V. Chopra, D. Ratz, L. Kuhn, T. Lopus, A. Lee, and S. Krein, "Peripherally inserted central catheter-related deep vein thrombosis: contemporary patterns and predictors," Journal of Thrombosis and Haemostasis, vol. 12, no. 6, pp. 847-854, 2014.

[12] R. S. Evans, J. H. Sharp, L. H. Linford et al., "Risk of symptomatic DVT associated with peripherally inserted central catheters," Chest, vol. 138, no. 4, pp. 803-810, 2010.

[13] T. K. Liem, K. E. Yanit, S. E. Moseley et al., "Peripherally inserted central catheter usage patterns and associated symptomatic upper extremity venous thrombosis," Journal of Vascular Surgery, vol. 55, no. 3, pp. 761-767, 2012.

[14] J. F. Timsit, J. C. Farkas, J. M. Boyer et al., "Central vein catheterrelated thrombosis in intensive care patients: incidence, risks factors, and relationship with catheter-related sepsis," Chest, vol. 114, no. 1, pp. 207-213, 1998, http://journal.publications .chestnet.org/data/Journals/CHEST/21768/207.pdf.

[15] T. J. Wilson, D. L. Brown, W. J. Meurer, W. R. Stetler Jr., D. A. Wilkinson, and J. J. Fletcher, "Risk factors associated with peripherally inserted central venous catheter-related large vein thrombosis in neurological intensive care patients," Intensive Care Medicine, vol. 38, no. 2, pp. 272-278, 2012.

[16] A. Hingorani, E. Ascher, J. Hanson et al., "Upper extremity versus lower extremity deep venous thrombosis," The American Journal of Surgery, vol. 174, no. 2, pp. 214-217, 1997.

[17] D. A. Sander and J. F. Martin, "Autopsy proven pulmonary embolism in hospital patients: are we detecting enough deep vein thrombosis?" Journal of the Royal Society of Medicine, vol. 82, no. 4, pp. 203-205, 1989.

[18] C. S. Kitchens, "How i treat superficial venous thrombosis," Blood, vol. 117, no. 1, pp. 39-44, 2011.

[19] E. Mendoza and C. Lattimer, "Superficial vein thrombosis," in Duplex Ultrasound of Superficial Leg Veins, E. Mendoza, C. R. Lattimer, and N. Morrison, Eds., pp. 217-225, Springer, Berlin, Germany, 2014.

[20] H. Decousus, I. Quéré, E. Presles et al., "Superficial venous thrombosis and venous thromboembolism: a large, prospective epidemiologic study," Annals of Internal Medicine, vol. 152, no. 4, pp. 218-224, 2010.

[21] R. F. Chemaly, J. B. De Parres, S. J. Rehm et al., "Venous thrombosis associated with peripherally inserted central catheters: a retrospective analysis of the Cleveland clinic experience," Clinical Infectious Diseases, vol. 34, no. 9, pp. 1179-1183, 2002.

[22] J. D. B. Kooij, F. M. van der Zant, E. J. R. van Beek, and J. A. Reekers, "Pulmonary embolism in deep venous thrombosis of the upper extremity: more often in catheter-related thrombosis," The Netherlands Journal of Medicine, vol. 50, no. 6, pp. 238242, 1997.

[23] A. Palla, G. Rossi, F. Falaschi, L. Marconi, M. Pistolesi, and P. Prandoni, "Is incidentally detected pulmonary embolism in cancer patients less severe? A case-control study," Cancer Investigation, vol. 30, no. 2, pp. 131-134, 2012. 


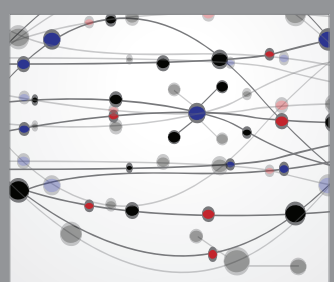

The Scientific World Journal
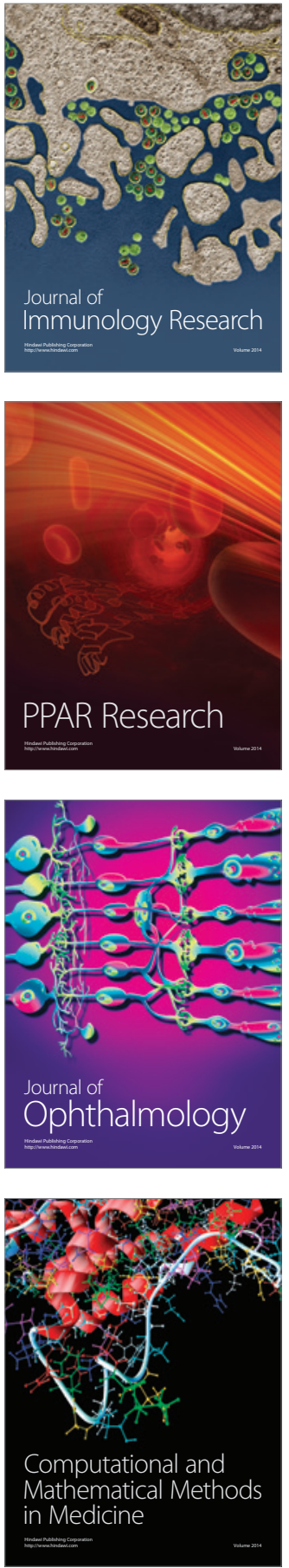

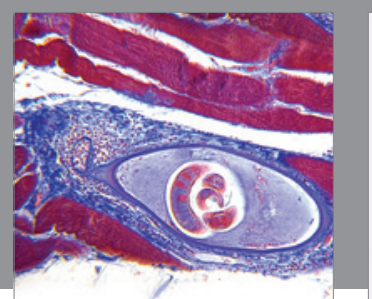

Gastroenterology Research and Practice

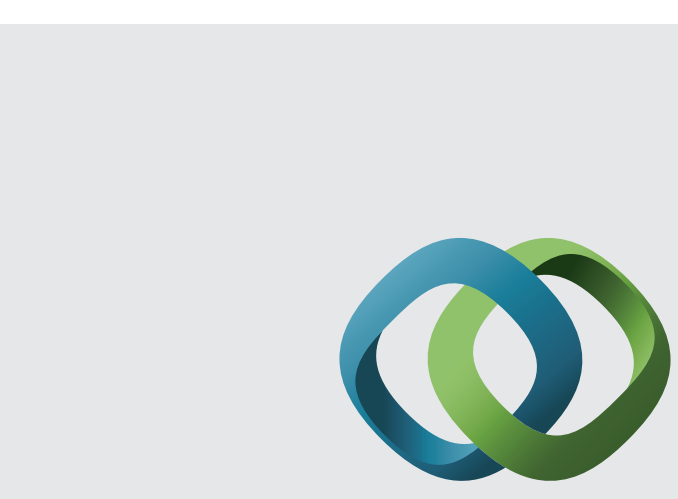

\section{Hindawi}

Submit your manuscripts at

http://www.hindawi.com
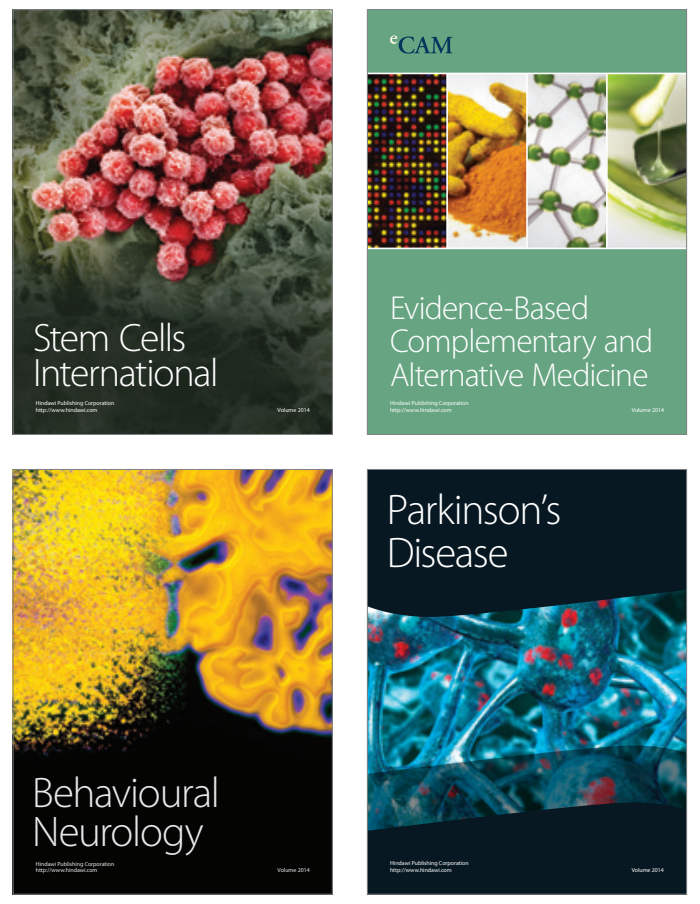
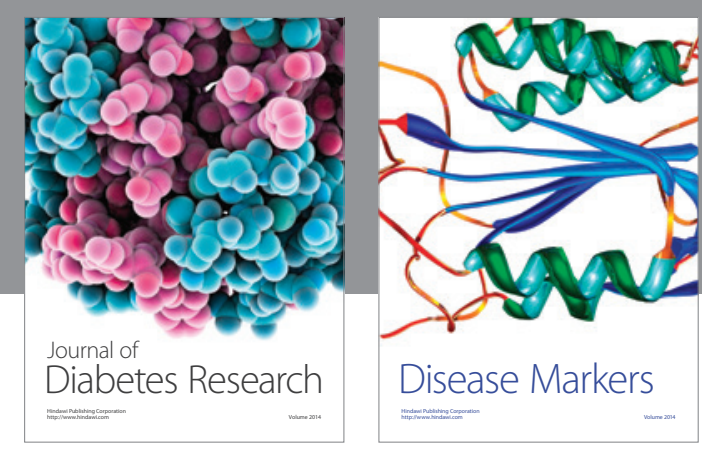

Disease Markers
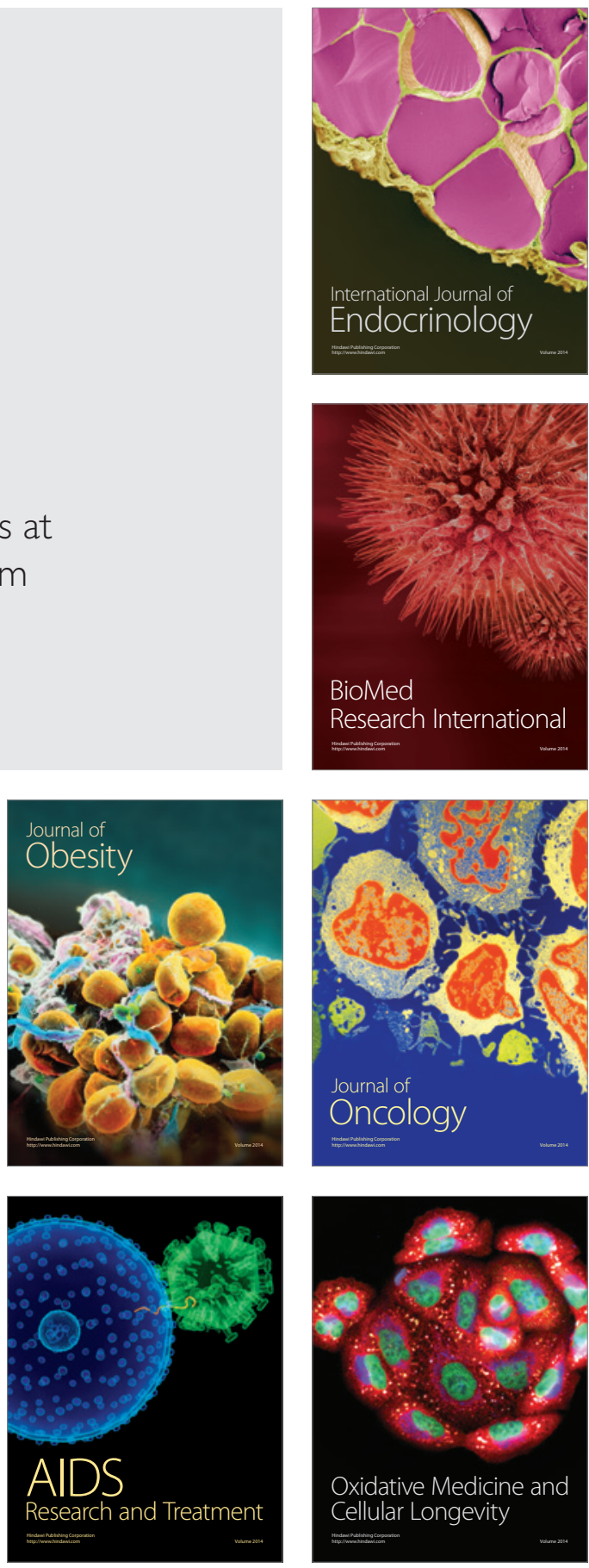